\title{
El Inca Titu Cusi Yupanqui, primer Cronista Peruano.
}

\author{
"es hombre de treynta y tres años \\ (Titu Cusi) muy bien entendido $y$ \\ alto',. \\ Juan de Matienzo \\ (Gobierno del Perú, 2.* parte, cap. 18)
}

En lo que sigue, trâtase únicamente de señalar la ubicación temporal, entre el grupo-de Cronistas peruanos-sin distinción de razas, - dejolìtepersonatyasobra del inca Titu Cusi Yupanqui (bautizado por el aguistino fray Juan de Vivero, con el nombre de Diego de Castro Titu Cusi Yupanqui, el 28 de agosto de I568), dejando para quien corresponda el estudio biográfico y la valoración específicamente histórica de su obra. Además, simultáneamente, se añade una bibliografía sumaria, que oriente en forma adecuada cualquier interés enrumbado hacia la persona y obra del penúltimo Inca peruano.

Cronológicamente, 1a "Instrucción del Inga don Diego de Castro Titu Cussi Yupangui para el Tlustre s'eñor el Licenciado Lope García de Castro, Governador que fué destos rreynos del Piru, tocante a los negocios que con su Magestad 
en su nombre por su poder ha de tratar", es la primera Crónica-entre las conocidas-que haya sido o escrito o dictada por un peruano. Este último viene a ser el caso del cusqueño Titu Cusi. El manuscrito estaba dirigido al monarca don Felipe II, por intermedio del licenciado Lope García de Castro (Gobernador del Perú, allá por los' años de $5_{564}$ a I569), relatándose las "cosas convenientes a mí y a mis subcesores" (pp. I09). Según esto, los fragmentos de la "Historia de los" Incas" del jesuita chachapoyano Blas Valera están en segundo lugar. E1 tercero, corresponde a la "Primera parte de los Comentarios Reales que tratan del origen de los' Incas, Reyes que fueron del Pirú, de su idolatría, leyes y gobierno en paz y en guerra", del cusqueño Garcilaso de la Vega Chimpuoillo. Se ubica en cuarto lugar la "Relación de Antiguedades deste Reyno del Piru', por Juan Santa Cruz Pachacuti, oriundo de la provincia de Tinta (Cusco). Por último, viene "El Primer Coronica i Buen Gobierno" de Felipe Huamán Poma de Ayala, nacido en la provincia de Lucanas (Ayacucho). Es curioso anotâp cónió cdichós' Cronistas peruanos no mencionan al inca Titu Cusi y si los extranjeros; aunque el padre de la Calancha, uno de los que más extensamente han escrito sobre el Inca, nació en la ciudad de Chuquisaca o La Plata, perteneciente por entonces a la juriśdicción del virreynato del Perú. A decir verdad, la omisión tiene diferentes causas.

Los fragmentos del padre Valera carecen de relación con el tema; y tampoco la tiene aquella Relación del Jesuita Anónimo, señalada por algunos autores como escrita por el jesuita chachapoyano. Garcilaso de la Vega omite, deliberadamente, por ser bastardo, la figura del Inca Titu Cusi, aunque Garcilaso era también un bastardo orgulloso de pertenecer a la auténtica nobleza incaica. La obra de Santa Cruz 
Pachacuti, llega solamente hasta la ocupación del Cusco por los invasores blancos. Finalmente, Huamán Poma de Ayala parece haber ignorado la existencia de Titu Cusi. Así, en su Crónica, foja 439, afirma que al salir Sayri Túpac de Vilcabamba dejó "en su lugar" a su hijo Túpac Amaru, errando además en la enunciación del parentesco entre Sayri Túpac y Túpac Amaru, hermanos sin lugar a dudas (véase "La descendencia de Huayna Cápac” por Ella Dúnbar Temp?e, Rev. Histórica, ts. XI-XIII, Lima I937-I940).

No existe problema acerca de la precedencia cronológica entre Titu Cusi y los tres Cronistas mencionados en último lugar. Por lo tanto, aquí se indicará únicamente lo que corresponda a Blas' Valera, con el propósito de eliminar enjuiciamientos inadecuados y ratificar lo enunciado en el título. $\mathrm{Y}$ antes de continuar, haciendo un paréntesis, se esboza una referencia acerca de las vicisitudes sufridas por la obra de Titu Cusi Yupanqui hasta el momento de su publicación en el año de I9I6.

El historiógrafbiblontefrafor costrinnénez de la Espada conoció el documento original, perteneciente a la Biblioteca del Escorial, dándole como título: "Relación de cómo los españo." les entraron en el Perú y el subceso que tuvo Mango Inga en el tiempo que entre ellos vivió". El año de I877, al publicar en Madrid la "Guerra de Quito", por Cieza de León, Jinénez de la Espada insertó un fragmento de la relación de $\mathrm{Ti}^{\star} u$Cusi,enelApéndiceI $8^{\circ}$, pp. I I 5 a 120 , es'cogiendo lo referente a la muerte del inca Manco II.

El erudito don Manuel González de la Rosa también conoció el documento inédito, sacando una copia y dándole como título: "Instrucción del Inga D. Diego de Castro Tito Cusi Yupangui para el muy ilustre señor Licenciado Lope García de Castro". Tuvo la intención de publicarla en uno de los tomos de su "Colección de Historiadores' del Perú", 
como lo manifiesta en la Introducción de la Historia de Lima del padre Cobo, pp. XIV, (Lima, I882), sin que pudiera lograr su propósito por la caótica situación en que se encontraba el Perú.

Posteriormente, don Carlos A. Romero recibió del señor González de la Rosa una copia, casi completa, del documento mencionado con anteroridad. Por transcripción enviada desde España logró entrar en posesión de las páginas que faltaban, publicando el documento íntegro en el tomo II de la "Colección de Libros y Documentos referentes a la Historia del Perí", precedida de una Biografía del Inca Titu Cusi Yupanqui. (El texto comprende II2 páginas. Los nueve Apéndices abrazan 37 páginas. $\mathrm{La}$ impresión fué realizada en la Imp. y Lib. Sanmarti, Lima, MCMXVI). Hasta aquí, la breve referencia sobre las yicisitudes' sufridas por la obra del Inca.

La crónica de Titu Cusi, a quien Jiménez de la Espada llama con propiedad "penúltimo de los monarcas naturales

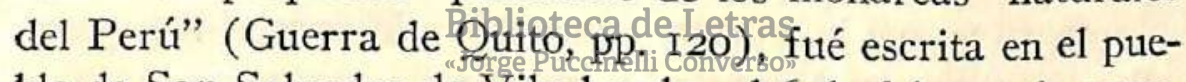
blo de San Salvador de Vilcabamba, el 6 de febrero de 1570 , dando fe de su autenticidad los sacerdotes agustinos fray Marcos García y fray Diego Ortiz y tres capitanes del Inca : Suya Yupanqui, Rimache Yupanqui y Sullca Várac (Instrucción, pp. I08 a 109). Explícitamente, el escribano y "lengua" Martín de Pando manifiesta haber puesto por escrito el relato de Titu Cusi "a insistion (sic) del dicho" Inca, relato previamente ordenado por fray Marcos García. Hay más. El inca Titu Cusi manifiesta, de manera clara, haber pedido el auxilio de dos personas conocedoras del idioma castellano y capaces de ordenar y escribir en forma correcta lo que declaraba para conocimiento del Monarca. Y así textualmente se lee en la página rog de su Instrucción: "rogué 


\section{, $-360-$}

al muy Reverendo Padre ffray iMarcos García y a Martín de Pando que confforme al usso de su natural, me ordenasen y compusiesen esta rrelación arriba dicha, para la enviar a los Reynos de España al muy Ilustre Señor el Licenciado. Lope García de Castro, para que por mi y en mi nombre, 1levando mi poder, me haga merced de la enseñar e rrelatar a su Magestad del Rey don Phelipe nuestro señor". Por otra. parte (olvidando la fecha de la Instrucción) se desprende del texto que cuando dicha obra fué terminada el gobernador Lope García de Castro había ya entragado el poder al virtrey' Toledo, quien entró en Lima a fines de noviembre de 1569 , y estaba próximo a partir para España. Así, en la Instrucción citada, pp. 3, refiriéndose al Licenciado dice textualmente: "Gobernador que fué destos rreinos.... que pués su Señoría va destos rreinos a los' de España". Más adelante, pp. 4, agrega: "que su Señorfa me haga merced, llegado que sea con bien a los rreynos de España, de dar a entender a su Magestad del Rey don Phelipe nuestro señor, debaxo de cuyo amparo yo me hipuesto guien isiey y la necesidad que a

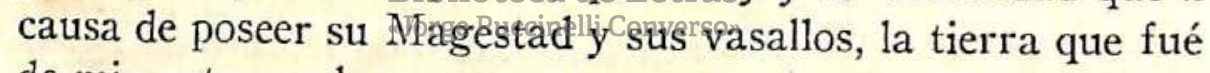
de mis antepasados en estos' montes padezco". En la página I08 de la obra citada se insiste en decir "Gobernador que ffue de estos Reinos", aludiendo al licenciado Castro. En la página rog Titu Cusi declara que la Instrucción se compuso para ser llevada y elevada ante el Monarca por el Licenciado. Por último, en el Poder otorgado por Titu Cusi, Ob. cit., pp. I Io, se dice de aquél: "que a los rreinos de España agora vá". En consecuencia, por la fecha en que fué terminado el documento ( 6 de febrero de 1570 ) y por las alusiones repetidas en la Crónica claramente aparece el inca Titu Cusi Yupanqui como el primer Cronista peruano, en contradicción con lo hasta aquí sostenido por el señor González de la Rosa, cur 


\section{$-3^{61}-$}

ya afirmación sobre la prioridad cronológica deł padre Valera había obtenido carta de casi indiscutida ciudadanía.

La Historia de los Incas del jesuita Blas Valera, escrita en elegante latín-según el decir de Garcilaso,-fué posterior a la Instrucción del inca Titu Cusi. A continuación van las pruebas de esta afirmación. Blas' Valera ingresó en el Colegio de jesuitas de Lima y fué recibido por el padre Jerónimo de Portillo el 20 de noviembre de I 568. Corría el año de I 57 I cuando pasó al Cusco con el fin de adoctrinar a los Naturales. El señor González de la Rosa (quien para loar con mayor comodidad a Valera consideró necesario denigrar, con los. epitetos más enojosos, la memoria del cronista Garcilaso) va. a ratificar, a pesar suyo, la bondad del aserto acerca de la precedencia temporal de Titu Cusi Yupanqui sobre su defendido el padre Valera. En tun artículo denominado "El padre Valera primer historiador pertuano", publicado en la Revista Histórica, tomo II, Foo \$ pp. I83, sostiene que es "segurísimo que reunió Valera sus materiales y redactó sus obras" con ocasión de Hiasibredripiezeraal Cusco ( 57 I o co-

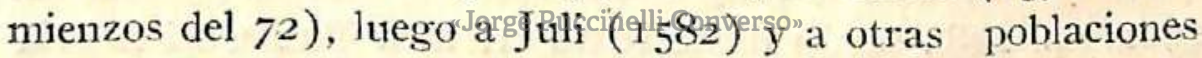
de la región del Titicaca.

El señor José Toribio Polo comparte la misma opinión, al considerarlo como el "primero, entre los nuestros, que quiso hacer conocer, con exactitud y verdad, el Perú antiguo, y el Imperio de los' Incas, destruido al empuje de las huestes españolas", en su artículo denominado "Blas Valera" (Revista Histórica, tomó II, entrega IV, Lima, I907, pp. 544).

Como lo que importa en el caso presente es, ante todo, la ubicación cronológica de la obra en su calidad de Crónica escrita por un Cronista peruano, se tratará de reforzar lo referente a la legitimidad de la fecha citada para la Instrucción de Titu Cusi ( 6 de febrero de 1570 ), mediante la mención de otros documentos que acrediten la situación $y_{s}$ reali- 
dad de los personajes que aparecen como testigos oficiales de la autenticidad del documento. Es curioso anotar cómo el señor González de la Rosa es a la vez defensor de la prioridad temporal de Valera y fracasado editor de una obra (la de Titu Cusi) que iba encaminada a desmentir su rotunda afirmación sobre la precedencia cronológica de aquél.

El momento más probable en que acaeció la muerte del Inca Titu Cusi Yupanqui, corrobora la autenticidad del documento. Es casi seguro que el Inca falleció en I571, es decir, un año después de escrita la Instrucción, pues cuando la gente enviada por el virrey. Toledo llegó a Vilcabamba y logró dominar aquella región y capturó al nuevo Inca Túpac Amaru, "hallaron que el ynca Titu Cusi Yupangui era muerto ya cerca avia de un año", según reza un "Inédito sobre el primer Túpac Amaru”, publicado por el señor Carlos A. Romero (Revista Históriea t. IT L ima, I907, pp. 70). Esto hecha por tierra una tardía afirmación contenida en las "Noticias cronológicas del Cuzeo" escritas' por don Diego de Es-

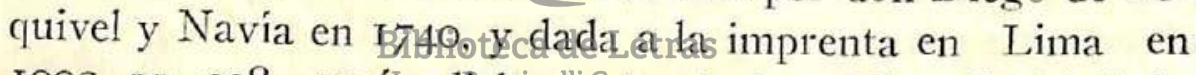

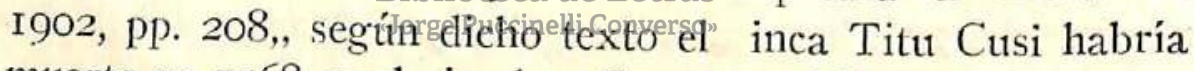
muerto en ${ }^{5} 58$, es decir, dos años antes del momento en que fué terminada la Instrucción tantas veces mencionada. Y contra tan peregrina afirmación existen otros' documentos, publicados por el señor Mackehenie, que más adelante mencionaremos. Por ahora, serán expuestas algunas ratificaciones sobre la existencia y ubicación de los testigos mencionados, para garantizar la veracidad de la obra del penúltimo Inca peruano refugiado en Vilcabamba.

El mismo día en que se terminó la instrucción ( 6 de febrero de ${ }_{5} 570$ ), ante el mismo escribano (Martín de Pando) y teniéndose como testigos a los mismos religiosos (los agustinos fray Marcos García y fray Diego Ortiz), el inca Titu Cusi otorgaba poder suficiente al licenciado Lope Gar- 
cía de Castro para que lo representase ante el rey don Felipe II; señalártose además la presencia de los testigos don $\mathrm{Pa}$ blo Guallpa Yupanqui, don Martín Cosi Guamán y don Gaspar Xulca Yánac.

En la Noticias Cronológica de Esquivel y Navía, pp. 208, el escribano Martin de Pando es calificado despectivamente como "mestizo apóstata, secretario del Inca". La persona de fray Marcos' está citada en dos cartas del Inca Titu Cusi, escritas en Pampacona en 6 de febrero y 24 de noviembre de 1568 (insertas por González de Barcia en el prólogo a la Florida del Inca, del cronista Garcilaso), dirigidas a fray Juan de San Pedro, provincial de la orden de San Agustín. La forma como el padre García se retiró de Vilcabamba (por discrepancias con Titu Cusi), está relatada por doña Angelina Llacsa Chuguri, quien recogió las noticias de labios de don Diego Ancalli, "cin indio capitán y de los más principales, que después quedó en el gobierno de la provincia y embalsamó el cuerpo del inga" (Col. de libros y documentos' referentes a la Histbliiatalel deeré, rserie I, tomo II, Apén-

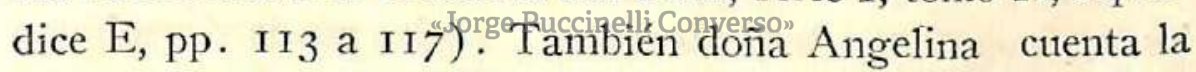
forma cómo murió en Vilcabamba fray Diego Ortiz. (Esta declaración fué tomada por don Carlos A. Romero del proceso original, encontrado en el Archivo Nacional de Lima, y formaba parte de uno de los tomos de manuscritos de la antigua Biblioteca Nacional).

El señor Carlos A. Mackehenie, en un artículo denominado "Apuntes sobre don Diego de Castro Titu Cusi Yupanqui”, escrito en la Rev. Histórica, t. III, trimestre IV, pp. 37 I a 390, y sin haber teído todavía la Instrucción de Titu Cusi, publicó los asientos del "Libro del Contador Miguel Sánchez, para el año de 1569 ", en donde se ordena pagar, el II de febrero y el 6 de diciembre de I 569 , al procura- 
dor del convento de San Agustín, fray Pablo Castro, la suma de 300 pesos de plata ensayada a nombre de fray Marcos' García, "a quien se deben de medio año que a doctrinado a Tito Cusi Yupangui Inga y a los yndios que con el están en 1a provincia de Vilcabamba". A continuación se dice algo parecido sobre fray Diego Ortiz, con fecha 18 de diciembre del mismo año (Art. citado, pp. $3^{8} 3$ ). Y en 5 de agosto de r 569 aparece un pago de 950 pesos' de plata, hecho a don Atilano de Anaya, apoderado del inca Titu Cusi Yupanqui, a cuenta de 2,000 pesos que mandó entregarle por una vez el gobernador Lope García de Castro (Art. cit. pp. $3^{8} 5$ ). Más tarde el señor Mackehenie rectificó algunas de sus afirmaciones en un nuevo artículo, bajo el mismo rubro que el anterior, y como consecuencia de haber leído la Instrucción del Inca (Rev. Histórica, tomo V, pp. 5 a I4). Lo anterior es una nueva comprobación de que-Titu Cusi vivió un tiempo mayor de lo que manifiéstan las Noticias Cronológicas de Esquivel y Navía.

Sobre la personalyiotatos de Titu Cusi Yupanqui con los representantes dénta Cononiaoespañola, puede consultarse la siguiente bibliografia sumaria:

I) Carta del licenciado Castro a S. M. con noticia de lo capitulado y concertado con el Inga rebelado..... en "Gobernantes del Perú. Cartas y papeles' del siglo XVI", tomo III, pub. por Roberto Levillier, Madrid I92I, pp. 263 a 269. 2) "Gobierno del Perú", del licenciado Juan de Matienzo, escrita antes de 1573 y dada a la imprenta en Buenos Aires el año de 1910 , cap. 18 $8^{\circ}$ pp. 193 a 198.3 ) "Relación del camino e viaje desde la ciudad del Cuzco a la tierra de guerra de 'Mango Inga....", por Diego Rodríguez de Figneroa, Corregidor que fué de Vilcabamba, publicada en Berlín el año rgro. 4) "Descripción y suscesos históricos' 
de la provincia de Vilcabamba", por Baltazar de Ocampo Conejeros, pub. por don Victor A. Maúrtua, en el tomo VII del Juicio de limites entre el Perú y Bolivia, impreso en Barcelona el año 1906, pp. 308 a 323. 5) "Una antigualla peruana", pub. en 1892 por don Marcos Jiménez de la Espada, y dado bajo el título de "Discurso sobre la descendencia y gobierno de los Incas", en la "Colección de libros y documentos referentes a la Historia del Perú", serie II, tomo III, pp. 3 a 53, conocida también como la Relación de los Quipucamayos a Vaca de Castro. 6) "“Virrey D. Francisco de Toledo", tomo VIII de la Colección Torres' de Mendoza, Madrid 1867 , capítulos XXVI al XXVII, pp. 263 a 269. 7) "Anales del Perú", por el clérigo Fernando de Montesinos, pub. por Víctor M. Maúrtua, tomo II, libr. II, pp. 44 a 45, Madrid igo6. Montesinos cita solamente a Titu Cusi y, sin hablar de Túpac Amaru, pasa a referir la captura del Inca por las tropas del virrey Toledo. 8) La Historia de los' Incas de Sarmiento de Gamboă, trae en su párrafo yo una bre-

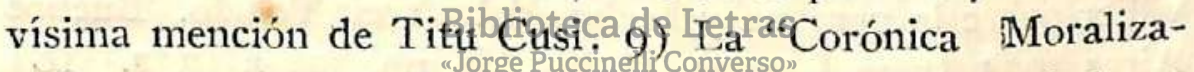
da" por el agustino fray Antonio de la Calancha, dada en Barcelona en I639, tomo I, lib. IV, caps. I al V, pp. 783 a 826 , es' una de las que se ocupa con mayor extensión del penúltimo Inca. Manifiesta el señor Carlos A. Romero que el proceso-roto en parte-sobre el martirio de fray Diego Ortiz, sacrificado a la muerte de Titu Cusi por el furor de los indígenas, pudo haber sido "destrozado, tal vez por la mano del agustino Calancha para escribir en su Crónica Moralizada los eapítulos referentes' a la muerte del mencionado sacerdote (véase la Biografía del Sr. Romero, Col. cit., serie I, tomo II) го) "Historia del Nuevo Mundo", por el p. Bernabé Cobo, pub. por Marcos Jiménez de la Espada. (Imp. E. Rasco. Sevilla r892), Lib. т2. cap. XXI, pp. 210 a 218. 
II) “Noticias cronológicas del Cusco", por Diego de Escuigo de Esquivel y Navía, Lima, 1902, pp. 206 a 209.

Entre posteriores estudios, aparte del efectuado por el señor Mackehenie, es necesario citar: 12) una breve Nota en "La Historia en el Perú" por el señor José de la Riva Agüero, Lima I9ıo, pp. 2I5 a 216. I3) Biografía por el señor Carlos A. Romero, Col. cit., serie I, tomo II, I4) "Historia de las guerras de los últimos Incas peruanos contra el poder español" ( I 535-I572), por Rómulo Cúneo Vidal, Barcelona s-a, parte V, caps. XXIV a XXX, pp. 221 a 263. I5). "Los Incas del Perú", por Clemente R. Markham (trad. M. Beltroy, Lima, I920), cap. XVIII, Pp. 245 a 25I. I6)

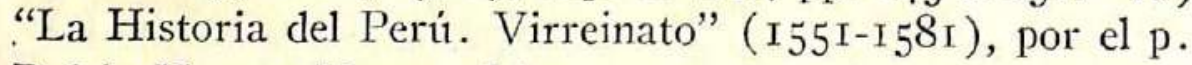
Rubén Vargas Ugarte, Lima, I935, Lección VIII, pp. 36 a 4I, Lección XIII, pp. 67 a 68 de "Historia del Perú. Vireynato" ( I55I-I590) por el mismo autor (Emp. "La Prensa", Lima 1942) - cap VHI, No. 5, pp. 91 a 92; cap. XIII, No. I, pp. I6r a 167 I7) La obra de don Roberto Levillier sobre la persona y hechos del virrey Toledo, Buenos

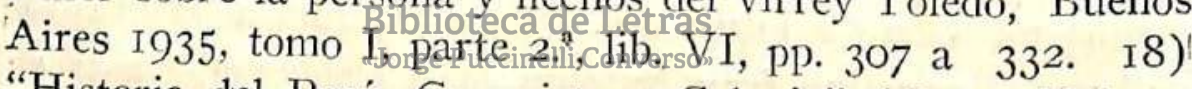
"Historia del Perú. Conquista y Colonia" (Curso Universitario), por don Raúl Porras (Lima I945), pp. 43 a 45.

En conclusión, de lo anterior se desprende la confirmación de lo manifestado al comienzo del presente artículo: que en una Historia de la Historia peruana el inca Titu Cusi Yupanqui aparece cronológicamente como el primer Cronista peruano, y su "Instrucción" como la primera obra que puede recibir el título de Crónica en forma objetivamente válida, según lo ratifican, directa e indirectamente, las sucintas referencias ya mencionadas.

Carlos Valcárcel. 